Article

\title{
A Force Measurement Method Based on Flexible PDMS Grating
}

\author{
Chengyu Jin ${ }^{1,2}$, Cui Ma ${ }^{1,2}$, Zhile Yang ${ }^{1}$ and Hui Lin ${ }^{1,2, * \mathbb{D}}$ \\ 1 Shenzhen Institutes of Advanced Technology, Chinese Academy of Sciences, Shenzhen 518055, China; \\ cy_jin1@126.com (C.J.); cui.ma@siat.ac.cn (C.M.); zl.yang@siat.ac.cn (Z.Y.) \\ 2 Shenzhen Key Laboratory of Precision Engineering, Shenzhen 518055, China \\ * Correspondence: hui.lin@siat.ac.cn
}

Received: 22 February 2020; Accepted: 25 March 2020; Published: 27 March 2020

\begin{abstract}
With the rapid development of flexible materials, various high-performance biocompatible flexible sensors have been proposed for specific measurement applications. Among these materials, polydimethylsiloxane (PDMS) is one of the most popular polymers by curing the mixture of pre-polymer (base) and cross-linker (curing agent). In this paper, a force measurement method based on PDMS grating is introduced. The PDMS grating is cast from a commercial master grating, which is precise, low-cost, and easy to follow. The elastic modulus can be controlled by the curing temperature and the mixing ratio. The PDMS grating is tested using a tension testing machine. As the stretching force increases, the grating line-spacing simultaneously increases and the diffraction light spot shifts. By capturing the light spot shift using a camera, the relationship between light spot position and stretching force is established and evaluated. Experimental results show that the linearity $\left(R^{2}\right)$ of the proposed method is better than 0.998 , adding that the sensitivity is $\sim 0.5-0.7 \mathrm{~N} / \mathrm{mm}$ and the accuracy is up to $0.05 \mathrm{~N}$.
\end{abstract}

Keywords: PDMS; elastic modulus; diffraction grating; force measurement

\section{Introduction}

With the development of industrial technology, various sensors have been invented to tackle various measurement problems. The majority of the traditional sensors are based on metal and semiconductor materials, of which tensile and sensitivity are limited and not suitable for detecting human activities. Compared with the traditional sensors, the flexible sensors overcome the shortcoming of brittleness and possess advantages in biocompatibility, stretchability, wearability and continuous detection [1]. Based on these unique characters, flexible sensors have been well adopted in the areas of electronic skin, robots, the bionic implants, man-machine interaction systems, as well as other artificial intelligence equipment $[2,3]$.

As the basis of flexible sensors, flexible materials have also attracted attention. Generally speaking, flexible materials include nanoparticles, nanowires, carbon nanotubes, graphene, organic materials, and so on [4]. At present, the most widely used flexible materials are polymers, which belong to the category of organic materials. The hardness of polymers is between the solid materials (e.g., metal, ceramics) and the liquid materials (e.g., hydrargyrum), resulting in a good comparability to human skin [5]. The most common types of polymer include PDMS (polydimethylsiloxane), PEN (polyethylene glycol terephthalate), PI (polyimide), PEI (polyetherimide), and PET (polyester). PI has high tensile strength and can be continuously used under high temperature. The elongation of PET can reach up to $150 \%$, and it is therefore suitable for particular applications such as artificial organs [6]. Compared with the above materials, PDMS has better flexibility and it is usually used as a polymer substrate. Combined with the MOEMS (micro optical electronic mechanical system) 
technique, PDMS has been used in various flexible electronic products, including nodes in the internet of things, medical equipment, solar cells, smart watches, and other devices [7].

One of the most important applications of PDMS is the flexible force sensor. In recent years, a variety of works on electronic sensors have been published, where the force signal is transferred to an electronic signal (e.g., capacitance, resistance, voltage). Viry et al. [8] designed a flexible three-dimensional tactile sensor based on PDMS to simulate natural touch. The author measured the value of force by collecting the change of capacitance's value. Vogt et al. [9] proposed a soft force sensor using micro-fluidic channels filled with conductive liquid. The normal and in-plane forces can be detected by measuring the electrical resistance changes of the channels when compressed. Lee et al. [10] presented a flexible piezoelectric sensor to measure the shear and normal forces by adapting polyvinylidene fluoride (PVDF) and three-dimensionally structured PDMS. Meanwhile, optical sensors are also developed for force measurement in specific cases. Cho et al. [11] presented a PDMS sensor for measuring fingertip force based on optical waveguide. When the sensor is deformed under applied force, the transmission modes in the PDMS waveguide change, so that the force can be derived by measuring the light intensity loss of the waveguide. In addition, Guo et al. [12] developed a strain measurement method based on PDMS grating. When the sample is stretched, the strain can be calculated by measuring the position shift of light spot due to the grating diffraction. However, the shape of the grating pattern made by oxygen treatment is not easy to precisely control.

In this study, a force measurement method based on PDMS grating is presented. The PDMS grating is fabricated using the soft casting method, which is precise and easy to follow. On the basis of previous studies [12], this paper not only introduced the fabrication and force sensing principle of the PDMS grating, but also investigates the property control of the force sensor by adjusting the curing temperature and the mixture ratio of the PDMS. The rest of this paper is organized as follow: Section 2 introduces the property of PDMS and fabrication of PDMS grating. Section 3 presents the force measurement principle and experimental setup. Section 4 demonstrates the experimental results, followed by Section 5 that concludes the paper.

\section{Property of PDMS and fabrication of PDMS grating}

In this section, the property of PDMS is introduced, and the process of making flexible gratings is shown step by step.

\subsection{Property of PDMS}

The material studied in this paper is polydimethylsiloxane (PDMS), which is a silicon-based polymer. This material is optically transparent and generally considered inert, non-toxic and non-flammable [13]. PDMS has the characteristics of elasticity and adjustable elastic modulus. The brief formula of PDMS is shown in Figure 1.

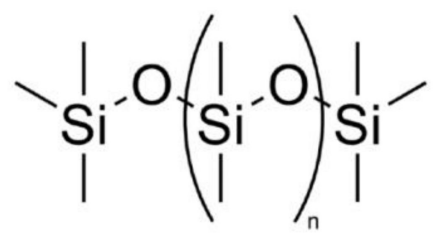

Figure 1. PDMS chemical formula.

Generally, PDMS is composed of an elastomer base and elastomer curing agent. By increasing the proportion of curing agent, the cross-linking structure of the material increases, leading to reduction of the elasticity and raise of the hardness. Moreover, heating treatment accelerates the cross-linking reaction [14]. In 2011, Wang et al. applied macro compression and nano indentation technology to measure the mechanical properties of PDMS [15]. In recent years, the most widely used mixing ratio 
of PDMS is 10:1, that is, 10 Sylgard 184 silicone elastomer base and 1 Sylgard 184 silicone elastomer curing agent [16].

Elastic modulus is an important property of PDMS, which is the quotient of stress to strain. Tensile test determines the strength and plasticity feature of a material by the relationship between stress and strain [17]. The first stage in the tensile test is so-called elastic deformation where the sample deforms linearly with the stress. With the rise of stress until the sample is yielded, the plastic deformation will occur. The following stage is the strain hardening, where the increasing stress leads to further deformation. Finally, the sample breaks and the stress is called fracture stress [18]. Particularly, the proposed force sensor works in the elastic deformation stage, where the elastic modulus determines the linear relationship between stress and strain.

To measure the elastic modulus of PDMS samples under different processing parameters, a tension tester is often used. The stress in the stretching direction is the ratio of tension force to cross-sectional area of the sample $[19,20]$. Meanwhile, the strain in the stretching direction is the ratio of tension displacement to the length of the sample. The elastic modulus of the PDMS grating sample can be calculated according to the stress and strain data recorded [21].

\subsection{PDMS Grating Fabrication}

Dow Corning Sylgard 184 silicone elastomer base (Sylgard 184A) and Sylgard 184 silicone elastomer curing agent (Sylgard 184B) are used to fabricate PDMS grating.

First, the electronic scale (BSA124S, Sartorius) is applied to weighting materials, and three solutions are prepared in mixing ratios of 5:1, 10:1 and 15:1 (Sylgard 184A to Sylgard 184B in weight) respectively.

Second, to make sure the material is evenly mixed, it is necessary to seal the glass bottle and evenly shake it up by a vortex mixer (Vorte2). Then, the glass bottle is put into a vacuum dryer (DZF-6020) for vacuum treatment for $20 \mathrm{~min}$, through which the bubbles in the material are completely removed.

Third, a commercial diffraction grating (\#48-462, Edmund Optics) with a groove density of 600 line/mm and a size of $50 \times 50 \mathrm{~mm}$ is selected as the master grating. The solution is poured evenly on the master grating and spin coated. The spin coater (KW-A4) rotates at 200r/min for 20s and at $500 \mathrm{r} / \mathrm{min}$ for $15 \mathrm{~s}$. After that, put the samples in the vacuum drying oven (DZF-6020) for heating, of which curing temperatures are set to the $70^{\circ} \mathrm{C}, 80^{\circ} \mathrm{C}$, and $90^{\circ} \mathrm{C}$ respectively for $3 \mathrm{~h}$.

Finally, take the samples out from the vacuum drying oven, and a PDMS film cast with grating structure is obtained. For easily mounting on the tension tester, the sample is cut into a shape of $50 \times 27 \mathrm{~mm}$ (length $\times$ width) by a fixture and a knife, and the dimension error is $\sim \pm 0.5 \mathrm{~mm}$. The thickness is $0.91 \pm 0.05 \mathrm{~mm}$ measured by a Vernier caliper (VOGEL 201035). It is worth noting that the thickness can be adjusted by changing the speed and time of spin coating.

The whole fabrication process of the PDMS grating is shown in Figure 2.

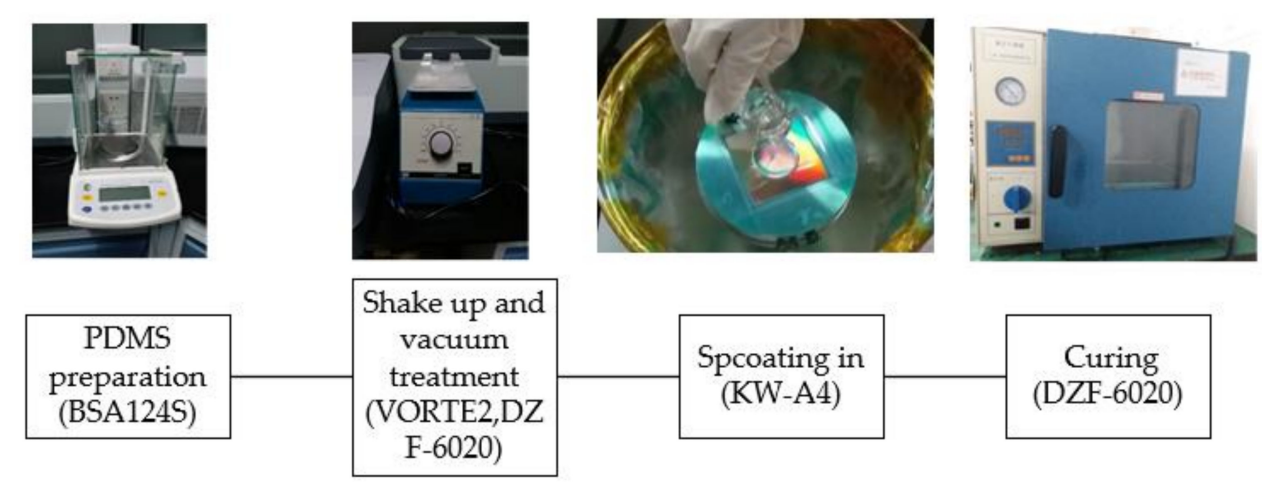

Figure 2. The fabrication process of flexible PDMS grating.

The PDMS grating is shown in Figure 3a and its micro pattern is observed by SEM (scanning electron microscope), as shown in Figure $3 b$. 


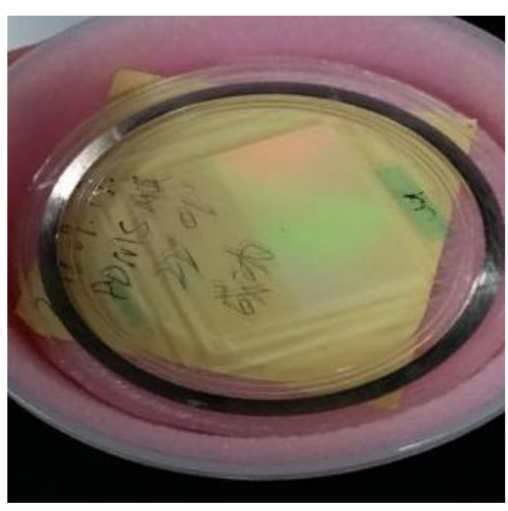

(a)

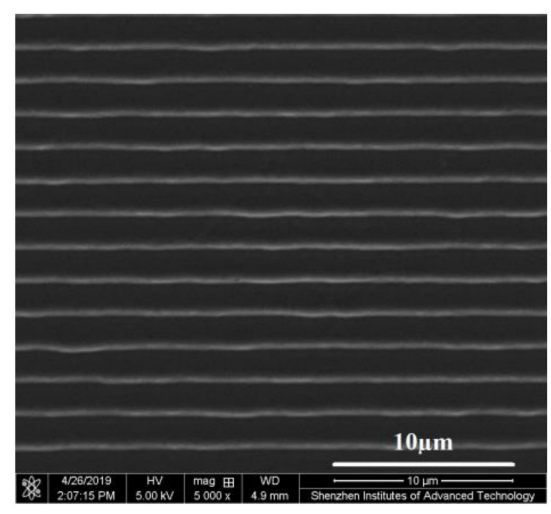

(b)

Figure 3. The PDMS grating profile: (a) camera photo (b) micro pattern observed by SEM

In this paper, three different mixing ratios and three different curing temperatures are chosen as the processing parameters, and nine sets of PDMS grating samples are prepared and tested.

\section{Force measurement}

\subsection{Measurement Principle}

The force measurement method is based on the diffraction property of the grating where the diffraction spot position can be calculated by the grating equation [22]. As shown in Figure 4, the laser light is normally incident to the PDMS grating, and the diffraction light spots are projected on to a screen with coordinate mark. The PDMS grating is stretched with an external force perpendicular to grating line. When the stretching force increases, the grating line-spacing simultaneously increases and the diffraction light spot shifts. By capturing the light spot shift using a camera, the relationship between light spot shift and stretching force can be established.

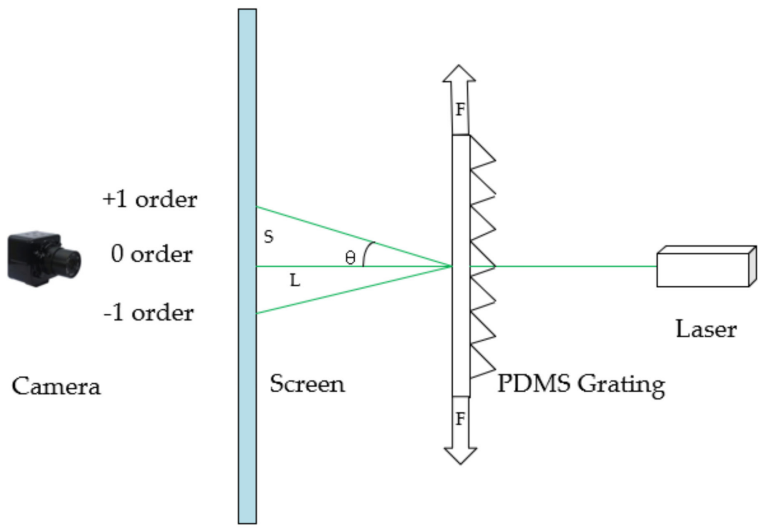

Figure 4. Force measurement principle based on PDMS grating.

First, according to the grating equation, the relationship of the grating line-spacing and laser wavelength $\lambda$ is denoted as,

$$
d \sin \theta=m \lambda=\lambda
$$

where $d$ : grating line-spacing; $\theta$ : diffraction angle; $\mathrm{m}$ : diffraction order-here the $1^{\text {st }}$ order is considered $(\mathrm{m}=1) ; \lambda$ : laser wavelength.

Second, from Figure 4, the geometric relationship is shown as follows,

$$
s=L \tan \theta
$$


where $L$ : the distance between the PDMS grating and the screen; $s$ : the position of the $1^{\text {st }}$ order light spot on the screen.

Third, the deformation of the PDMS grating follows Hooke's law as it is regarded as an ideal elastic material,

$$
E=\sigma / \varepsilon
$$

where E: the elastic modulus; $\sigma$ : the stress; $\varepsilon$ : the strain.

The stress $\sigma$ is calculated by,

$$
\sigma=F / A
$$

where $A$ : cross-sectional area of the sample; $F$ : force in the direction of tension.

As the grating is homogeneous, the strain $\varepsilon$ of the sample is equal to the extension ratio of each grating line,

$$
\varepsilon=\Delta d / d
$$

where $\Delta d$ denotes the variation of the grating line-spacing.

From Equations (3), (4), and (5), the following relationship can be derived,

$$
\frac{\Delta d}{d}=\frac{F}{E A}
$$

Fourth, infinitesimal analysis of Equation (1) and (2) is done,

$$
\begin{aligned}
& \cos \Delta \theta=-\frac{\lambda}{d^{2}} \Delta d \\
& \Delta s=\frac{L}{\cos ^{2} \theta} \Delta \theta
\end{aligned}
$$

where $\Delta \theta$ : the variation of the diffraction angle; $\Delta s$ : the light spot shift.

Finally, the relationship of $\mathrm{F}$ and $\Delta s$ can be derived as follows,

$$
F=-E A \frac{d \cos ^{3} \theta}{\lambda L} \Delta s
$$

It is easy to find that the tension force $F$ is proportional to the light spot shift $\Delta s$, as long as $\Delta s$ is relatively small. In other words, $F$ can be linearly derived by measuring the light spot position.

\subsection{Experimental Setup}

The experimental setup is built as shown in Figure 5. A 532nm DPSS laser ( $85 \mathrm{~mW}, \mathrm{CNI})$ is used as the light source. The line-spacing of the PDMS grating is $1.67 \mu \mathrm{m}$ (600 line/mm). The sample size is $50 \times 27 \times 0.91 \mathrm{~mm}$ (length $\times$ width $\times$ thickness) and the cross-sectional area is $24.57 \mathrm{~mm}^{2}$. A tension testing machine (HKE-3511) is applied for tension force loading. The distance between grating and screen is $94.5 \mathrm{~mm}$; A CCD camera (DMK23G445) of $1280 \times 960$ pixels is used for taking photos of the diffraction light spots. 


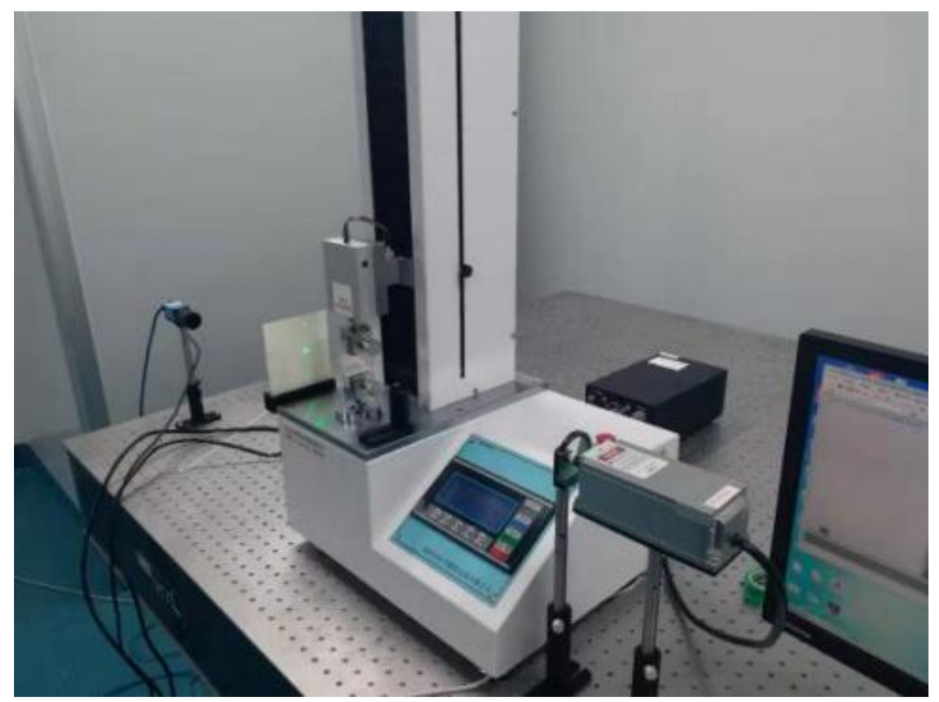

Figure 5. The experimental setup for force measurement.

The light spots captured by the CCD camera are shown in Figure 6a, where the light spot represents $+1^{\text {st }}, 0$, and $-1^{\text {st }}$ diffraction order from top to bottom respectively. It is crucial to determine the positions of the light spots. In the light of this, a MATLAB tool based on image binarization and center extraction algorithm is developed to process the image [23]. After processing, the center of the light spot can be found and the coordinate data can be derived, as shown in Figure $6 \mathrm{~b}$.

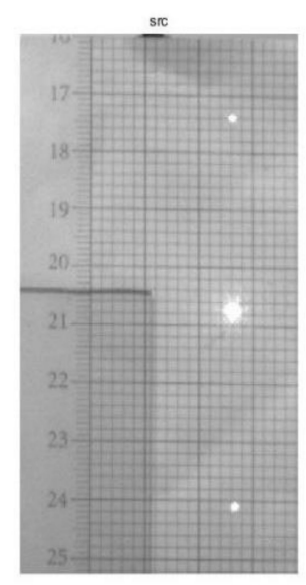

(a)

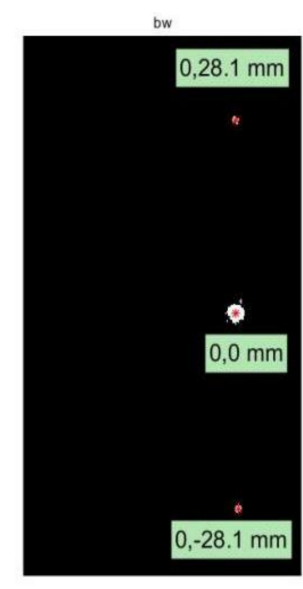

(b)

Figure 6. Image processing for light spot position determination by MATLAB, (a) original image, (b) processed image.

\section{Results}

\subsection{The elastic modulus}

Figure 7 illustrates the test results of elastic modulus under nine different sets of mixing ratios and curing temperatures. Ten samples are prepared for each set of mixing ratio and curing temperature, and each data point in Figure 7 is average of the 10 samples. 


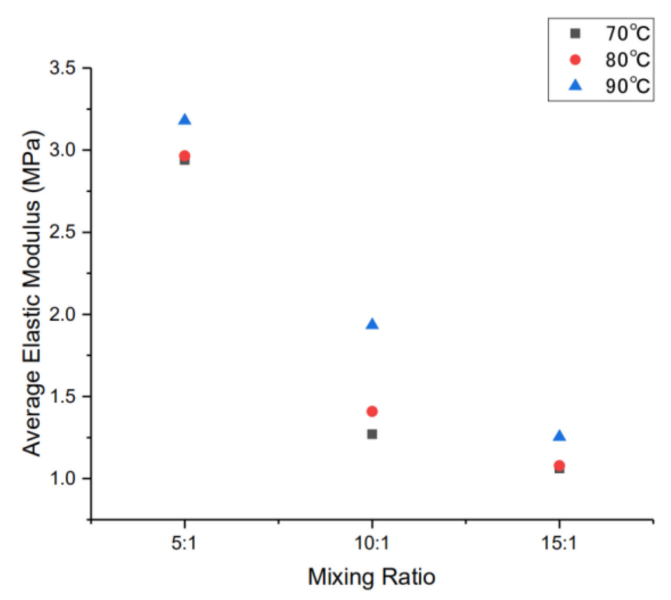

Figure 7. The average data of elastic modulus under different mixing ratios and curing temperatures.

On one hand, when the curing temperature is fixed, the elastic modulus under 5:1 mixing ratio is the maximum and the 15:1 one is the minimum. That is, the elastic modulus of PDMS decreases with the rise of proportion of elastomer base, whereas the relationship is not strictly linear.

On the other hand, when the mixing ratio is fixed, the elastic modulus increases with the rise of the temperature in the range of $70-90^{\circ} \mathrm{C}$. However, the results under $70^{\circ} \mathrm{C}$ and $80^{\circ} \mathrm{C}$ are close, whereas the result under $90^{\circ} \mathrm{C}$ is significantly higher. Therefore, the relationship of elastic modulus and curing temperature is also not strictly linear.

To investigate the repeatability of the fabrication process, the data distribution of the elastic modulus under curing temperature of $80^{\circ} \mathrm{C}$ is chosen for the illustration in Figure 8. The data deviation under mixing ratio of 5:1 is much larger than those under 10:1 and 15:1. That is to say, the precise control of elastic modulus is difficult using the mixing ratio of 5:1, and PDMS gratings with mixing ratios of 10:1 and 15:1 are chosen for further force measurement.

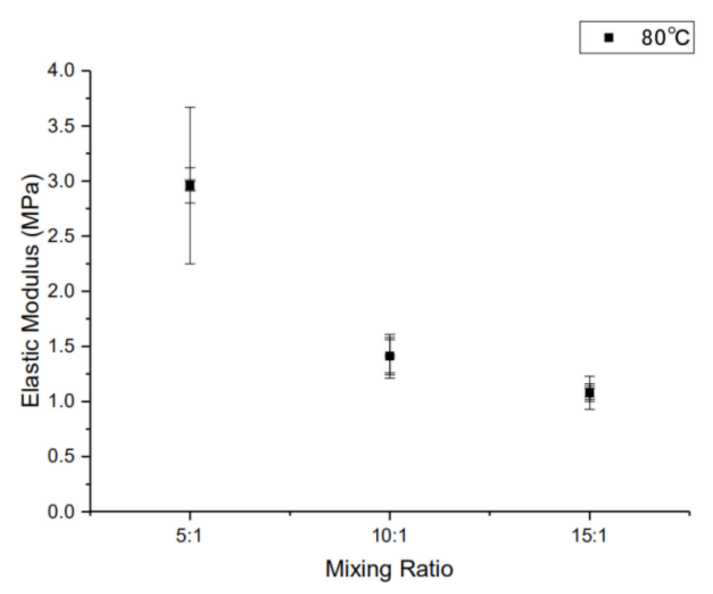

Figure 8. The data distribution of elastic modulus along with the mixing raito. (Curing temperature $=80^{\circ} \mathrm{C}$ )

\subsection{Force Measurement}

Two PDMS grating samples with mixing ratios of 10:1 and 15:1 are tested respectively. For the 10:1 samples, the processed photos of light spots are shown in Figure 9. The accurate position of each light spot is calculated and illustrated. It is obvious that the spots move closer with the increase of force. 


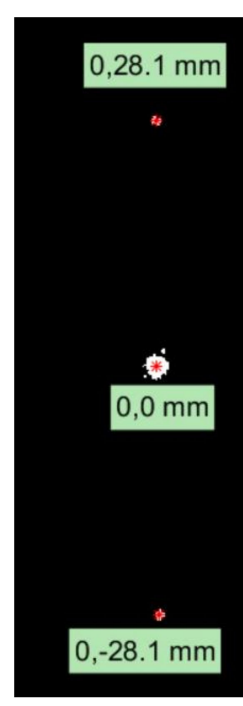

(a)

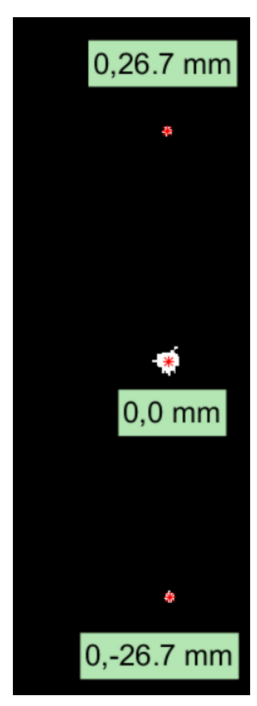

(b)

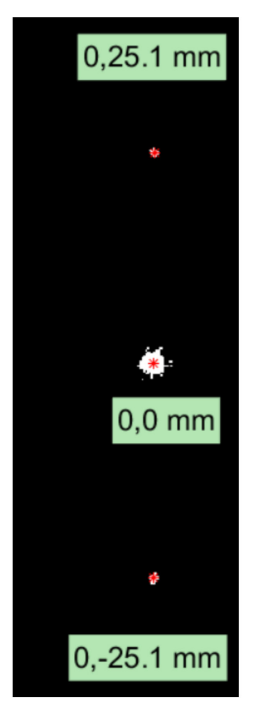

(c)

Figure 9. Images of light spot position under different forces: (a) $F=5 \mathrm{~N},(\mathbf{b}) \mathrm{F}=7 \mathrm{~N},(\mathbf{c}) \mathrm{F}=10 \mathrm{~N}$.

The data series of the light spot position and the corresponding tensile force are analyzed using linear regression method. The fitting curves are shown in Figure 10.

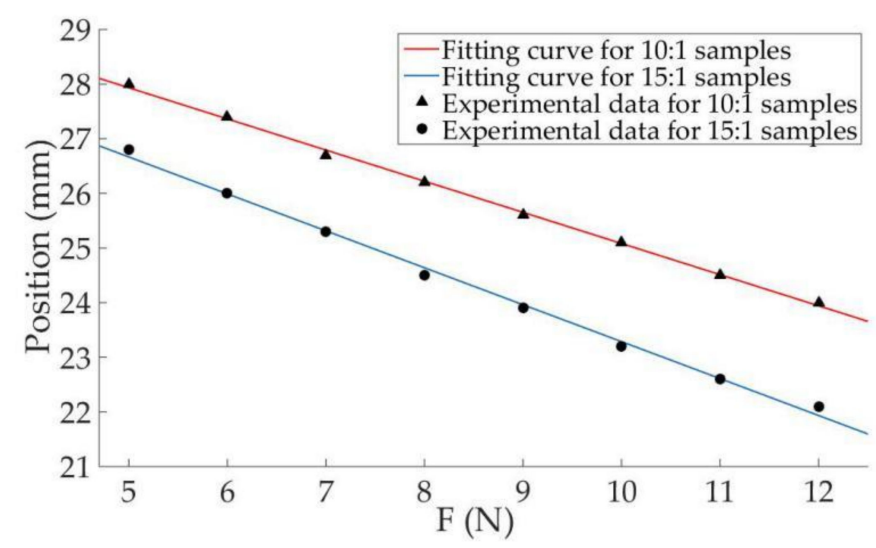

Figure 10. The experimental data and fitting curve of the light spot position and tensile force.

The discussion on Figure 10 are listed as below,

(1) Linearity. The coefficient of determination $\left(\mathrm{R}^{2}\right)$ for the $10: 1$ sample is 0.9999 , and that for $15: 1$ sample is 0.9985 . Therefore, the relationship between light spot position and tensile force is linear, which coincides with Equation (9).

(2) Sensitivity. The slope for the red line (10:1 sample) is $-0.57 \mathrm{~mm} / \mathrm{N}$, while the slope for the blue line (15:1 sample) is $-0.67 \mathrm{~mm} / \mathrm{N}$. The $15: 1$ sample has larger sensitivity than the $10: 1$ one. As illustrated in Figure 10, the elastic modulus is $\sim 1.5 \mathrm{Mpa}$ for 10:1 mixing ratio and $\sim 1.1 \mathrm{Mpa}$ for 15:1 mixing ratio. Therefore, the 15:1 sample has larger deformation than the 10:1 sample under the same external force, leading to a larger shift of the light spot. Based on this, the sensitivity of the PDMS grating force sensor can be adjusted by changing the mixing ratio, or even the cured temperature.

(3) Accuracy. The residual error of linear regression is used to evaluate the accuracy of force measurement. The mean residual error of the data points in Figure 10 is $0.05 \mathrm{~N}$ and the maximum error is $0.09 \mathrm{~N}$, which is sufficiently precise for force measurement. The error mainly comes from the uncertainty of spot position measurement, which is $\sim 0.05 \mathrm{~mm}$. According to the sensitivity analysis above, $0.05 \mathrm{~mm}$ error in spot position leads to $\sim 0.03 \mathrm{~N}$ error in force measurement. 
(4) Range. The force data in Figure 10 is ranging from $5 \mathrm{~N}$ to $12 \mathrm{~N}$. However, the maximum range is related with the fracture force of the sample. The fracture force is $\sim 30 \mathrm{~N}$ by a series of experiments. It can be estimated that linear and reliable results can be obtained using this method when the force is less than $20 \mathrm{~N}$.

The force sensor based on PDMS waveguide in [11] has linearity (R2) of 0.96 , error of $\sim 0.3 \mathrm{~N}$, and range of $5 \mathrm{~N}$. The proposed force sensor based on PDMS grating has a larger footprint than [11], and possesses better performance. Also using a PDMS grating, the relative error of strain measurement in [12] is less than $0.6 \%$. In this paper, the average relative error is estimated as $0.59 \%(0.05 \mathrm{~N} / 8.5 \mathrm{~N}, 8.5 \mathrm{~N}$ is the average force in the test), which is comparable with this key factor in [12]. However, the proposed soft casting method in this paper does not require any special equipment, and it is even cheaper and easier than the oxygen treatment method in [12]. Moreover, the PDMS grating pattern can be faithfully replicated from the master grating, which is favorable for mass production.

\section{Conclusions}

In this paper, a force measurement method based on PDMS grating is proposed. The fabrication of PDMS grating and property of PDMS are also introduced in detail. The following conclusions are drawn:

1. The PDMS grating is cast from a commercial grating, and the fabrication process is easy to follow.

2. The measurement principle is transferring the force signal to the position shift of diffraction light spot, an optical signal. The relationship between light spot position and tensile force is linear $\left(\mathrm{R}^{2}>0.9980\right.$ ). The sensitivity is $\sim 0.5-0.7 \mathrm{~mm} / \mathrm{N}$ (mixing ratio $10: 1$ and $15: 1$, curing temperature $80^{\circ} \mathrm{C}$ ) and the accuracy is up to $0.05 \mathrm{~N}$.

3. Elastic modulus is one of the most important characters for PDMS. It is negatively correlated with the mixing ratio (Sylgard 184A to Sylgard 184B in weight), and positively correlated with the curing temperature. One can control the sensitivity of the proposed force sensor by tuning the elastic modulus.

PDMS material has been widely used in the current research on flexible devices due to its excellent elastic property, good biocompatibility, and adaptability to the light. The proposed method based on PDMS grating is an optical sensing method, and therefore has potential for various specific force measurements in high electromagnetic interference applications. Moreover, the proposed method can be used to study the mechanical property of flexible material itself. This method is not only suitable for the uniform deformation measurement in this paper, but can also be extended to study non-uniform deformation such as large bending. By measuring different points on the sample surface, the stress/strain distribution can be obtained.

A possible improvement of the proposed method is using reflection grating instead of the transmission grating. At the reflection mode, the sensor is easier to package and more suitable for measuring opaque sample. The challenge is coating the PDMS surface with smooth metallic reflective film, which needs further research efforts in the future.

Author Contributions: H.L. contributed to the main idea of this paper; C.J. performed the experiments, analyzed the data and wrote the paper; C.M. wrote the image processing codes; Z.Y. and H.L reviewed and edited the paper. All authors have read and agreed to the published version of the manuscript.

Funding: The presented work is supported by the following Grants: National Natural Science Foundation of China Grant No. U1713210; Shenzhen Fundamental Research Program Grant No. JCYJ20170818163928953

Conflicts of Interest: The authors declare no conflict of interest.

\section{References}

1. Ramuz, M.; Tee, B.C.-K.; Tok, J.B.-H.; Bao, Z. Transparent, Optical, Pressure-Sensitive Artificial Skin for Large-Area Stretchable Electronics. Adv. Mater. 2012, 24, 3223-3227. [CrossRef] [PubMed] 
2. Han, S.T.; Peng, H.; Sun, Q.; Venkatesh, S.; Chung, K.S.; Lau, S.C.; Zhou, Y.; Roy, V.A.L. An overview of the development of flexible sensors. Adv. Mater. 2017, 29, 1700375. [CrossRef] [PubMed]

3. Chinnappan, A.; Baskar, C.; Baskar, S.; Ratheesh, G.; Ramakrishna, S. An overview of electrospun nanofibers and their application in energy storage, sensors and wearable/flexible electronics. J. Mater. Chem. C 2017, 5, 12657-12673. [CrossRef]

4. Mata, A.; Fleischman, A.J.; Roy, S. Characterization of polydimethylsiloxane (PDMS) properties for biomedical micro/nano systems. Biomed. Microdevices 2005, 7, 281-293. [CrossRef] [PubMed]

5. Hénot, M.; Drockenmuller, E.; Leger, L.; Restagno, F. Friction of Polymers: From PDMS Melts to PDMS Elastomers. ACS Macro Lett. 2018, 7, 112-115. [CrossRef]

6. Johnston, I.; McCluskey, D.; Tan, C.K.L.; Tracey, M. Mechanical characterization of bulk Sylgard 184 for microfluidics and microengineering. J. Micromech. Microeng. 2014, 24, 35017. [CrossRef]

7. Stojanović, G. Sensors and other electronic components on flexible substrates: From materials to applications. In Proceedings of the 2016 5th Mediterranean Conference on Embedded Computing (MECO), Bar, Montenegro, 12-16 June 2016; pp. 4-6.

8. Viry, L.; Levi, A.; Totaro, M.; Mondini, A.; Mattoli, V.; Mazzolai, B.; Beccai, L. Flexible Three-Axial Force Sensor for Soft and Highly Sensitive Artificial Touch. Adv. Mater. 2014, 26, 2659-2664. [CrossRef] [PubMed]

9. Vogt, D.M.; Park, Y.-L.; Wood, R.J. Design and Characterization of a Soft Multi-Axis Force Sensor Using Embedded Microfluidic Channels. IEEE Sens. J. 2013, 13, 4056-4064. [CrossRef]

10. Lee, Y.R.; Chung, J.; Oh, Y.; Cha, Y. Flexible Shear and Normal Force Sensor Using only One Layer of Polyvinylidene Fluoride Film. Appl. Sci. 2019, 9, 4339. [CrossRef]

11. Cho, H.; Lee, H.; Kim, Y.; Kim, J. Design of an optical soft sensor for measuring fingertip force and contact recognition. Int. J. Control. Autom. Syst. 2017, 15, 16-24. [CrossRef]

12. Guo, H.; Tang, J.; Qian, K.; Tsoukalas, D.; Zhao, M.; Yang, J.; Zhang, B.; Chou, X.; Liu, J.; Xue, C.; et al. Vectorial strain gauge method using single flexible orthogonal polydimethylsiloxane gratings. Sci. Rep. 2016, 6, 23606. [CrossRef] [PubMed]

13. Grzybowski, B.; Qin, N.; Haag, R.; Whitesides, G.M. Elastomeric optical elements with deformable surface topographies: Applications to force measurements, tunable light transmission and light focusing. Sensors Actuators A Phys. 2000, 86, 81-85. [CrossRef]

14. Liu, M.; Sun, J.; Chen, Q. Influences of heating temperature on mechanical properties of polydimethylsiloxane. Sensors Actuators A Phys. 2009, 151, 42-45. [CrossRef]

15. Wang, Z. Polydimethylsiloxane Mechanical Properties Measured by Macroscopic Compression and Nanoindentation Techniques. Ph.D. Thesis, University of South Florida, Tampa, FL, USA, 2011.

16. Dai, X.; Xie, H. New methods of fabricating gratings for deformation measurements: A review. Opt. Lasers Eng. 2017, 92, 48-56. [CrossRef]

17. Fincan, M. Assessing Viscoelastic Properties of Polydimethylsiloxane (PDMS) Using Loading and Unloading of the Macroscopic Compression Test. Ph.D. Thesis, University of South Florida, Tampa, FL, USA, 2015.

18. Walker, J. Halliday E Resnick Fundamentals of Physics; Wiley: Hoboken, NJ, USA, 2014.

19. Kishimoto, S.; Huimin, X.; Shinya, N. Electron moiré method and its application to micro-deformation measurement. Opt. Lasers Eng. 2000, 34, 1-14. [CrossRef]

20. Chen, J.; Zheng, J.; Gao, Q.; Zhang, J.; Zhang, J.; Omisore, M.; Wang, L.; Li, H. Polydimethylsiloxane (PDMS)-Based Flexible Resistive Strain Sensors for Wearable Applications. Appl. Sci. 2018, 8, 345. [CrossRef]

21. Khanafer, K.; Duprey, A.; Schlicht, M.; Berguer, R. Effects of strain rate, mixing ratio, and stress-strain definition on the mechanical behavior of the polydimethylsiloxane (PDMS) material as related to its biological applications. Biomed. Microdevices 2008, 11, 503-508. [CrossRef] [PubMed]

22. Palmer, C.A.; Loewen, E.G. Diffraction Grating Handbook; Newport Corporation: New York, NY, USA, 2005.

23. Pan, B.; Qian, K.; Xie, H.; Asundi, A. Two-dimensional digital image correlation for in-plane displacement and strain measurement: A review. Meas. Sci. Technol. 2009, 20, 62001. [CrossRef]

(C) 2020 by the authors. Licensee MDPI, Basel, Switzerland. This article is an open access article distributed under the terms and conditions of the Creative Commons Attribution (CC BY) license (http://creativecommons.org/licenses/by/4.0/). 